\title{
Síndrome hemorrágico por contacto con orugas del género Lonomia (Saturniidae) en Casanare, Colombia: informe de dos casos
}

\author{
Daniel Pineda ${ }^{1}$, Angela Amarillo ${ }^{2}$, Julio Becerra ${ }^{3}$, Gabriel Montenegro ${ }^{4}$ \\ ${ }^{1}$ Instituto de Salud en el Trópico, Facultad de Medicina, Universidad Nacional de Colombia, Bogotá D.C., \\ Colombia. \\ ${ }^{2}$ Fundación Nova Hylaea, Bogotá, D.C., Colombia. \\ ${ }^{3}$ Facultad de Medicina, Universidad Nacional de Colombia, Bogotá, D.C., Colombia. \\ ${ }^{4}$ Hospital San Carlos, Bogotá, D.C., Colombia. \\ El presente artículo describe los dos primeros casos de accidentes por orugas de Lonomia \\ diagnosticados en Colombia. Estos insectos ocasionan un cuadro local con alteraciones \\ hemorrágicas caracterizadas por una intensa actividad fibrinolítica y valores bajos de diferentes \\ factores de la coagulación. Los dos casos se presentaron en el departamento de Casanare y \\ fueron remitidos al Hospital San Carlos de Bogotá. La evolución de cada uno fue diferente: el \\ primero, con hemoptisis e insuficiencia renal secundaria, que le causó la muerte a la paciente; \\ el segundo, con gingivorragia y sangrado vaginal que evolucionó hasta la completa mejoría \\ después de la administración del suero anti-Lonomia. Descritos estos primeros casos, es \\ necesario ampliar el conocimiento sobre el tema y trabajar en su prevención.
}

Palabras claves: síndrome hemorrágico, fibrinolisis, oruga venenosa, Lonomia, Saturniidae, lepidóptera.

Hemorrhagic syndrome caused by Lonomia (Saturniidae) caterpillars in Casanare, Colombia: two cases

Two cases of contact with Lonomia caterpillars were diagnosed in Colombia. Lonomia caterpillars cause hemorrhagic disorders characterized by an intense fibrinolytic activity and low levels of several coagulation factors. Both cases occurred in the Casanare Department and were referred to San Carlos Hospital in Bogota. Each case developed differently. The first died as a consequence of hemoptisis and secondary renal failure, and the second had symptoms of gum and vaginal bleeding but recovered after administration of anti-Lonomia serum. These are first cases of Lonomia contact in Colombia; initiation of syndrome recognition, research, and prevention programs are recommended.

Key words: hemorrhagic syndrome, fibrinolysis, venomous caterpillar, Lonomia, Saturniidae, lepidoptera.

Desde 1967 se han informado accidentes por orugas del género Lonomia y de otras de la familia Saturniidae en Venezuela, Brasil, Perú, Guyana Francesa, Paraguay y Argentina. Las orugas de Lonomia al entrar en contacto con la piel del hombre, ocasionan una reacción local inicial y, de 12 a 24 horas después, un cuadro hemorrágico caracterizado por una intensa actividad fibrinolítica.

Correspondencia:

danielpineda@hotmail.com

Recibido: 21/12/00; aceptado: 31/08/01
También se ha descrito la insuficiencia renal aguda como complicación de los trastornos hemorrágicos $\mathrm{y}$, en ocasiones, con resultados fatales en Venezuela y Brasil (1-9).

Lonomia achelous y Lonomia obliqua, especies implicadas en este tipo de accidentes, no estaban descritas en Colombia pero sí en Ecuador, Perú, Brasil y Venezuela. Sin embargo, sí se habian descrito Lonomia descimoni en Guainía, Lonomia rufescens en el Valle del Cauca y Boyacá, Lonomia cluacina en el Valle del Cauca, Lonomia 
occidentalis en el Valle del Cauca, Caldas y Antioquia, Lonomia armata en Bogotá y Lonomia inexpectata en la ruta de Bogotá a Villavicencio (localidad no especificada) (10).

El veneno de las orugas es líquido y se inocula a la víctima al entrar en contacto con las cerdas de la oruga. El ingreso del veneno produce inicialmente un dolor intenso, seguido de malestar general, náuseas y cefalea; las manifestaciones hemorrágicas aparecen tardiamente, inicialmente con equimosis en el sitio del contacto seguido por sangrado gingival y, en ocasiones, epistaxis, sangrado vaginal, hematuria, sangrado digestivo y hemorragia endocraneana. En los exámenes paraclínicos, se encuentra una disminución de fibrinógeno, factor $\mathrm{V}$, factor XIII, plasminógeno y $\alpha$-2-antiplasmina, con recuento de plaquetas generalmente normal y aumento de los productos de degradación del fibrinógeno, factor von Willebrand y factor VIII:c. Otros factores como el II y el VII y la proteína $C$ se ven afectados de diferentes maneras $(2,8,11-18)$.

Los valores de fibrinógeno en los humanos pueden tardar hasta más de 20 días en normalizarse y están relacionados con la cantidad de veneno inoculado. Los efectos del veneno pueden variar según la especie implicada y el estado de desarrollo en el que se encuentre la oruga. En los accidentes por $L$. aquelous y $L$. obliqua, las pruebas de coagulación han mostrado prolongación de los tiempos de trombina, protrombina y parcial de tromboplastina $(8,14,16)$.

Se considera que en la fisiopatología del envenenamiento por $L$. achelous se superponen dos fenómenos. El primero es la intensa actividad fibrinolítica debida a la activación del sistema fibrinolítico y a la proteólisis del factor XIII; el segundo, por su parte, es una coagulación intravascular diseminada (CID) moderada debida a los agentes procoagulantes. Igualmente, se han identificado activadores de la protromina en el veneno de $L$. achelous, un activador termoestable del factor $\mathrm{V}$ y un inhibidor termolábil del factor $\mathrm{V}$, entre otros. Para el veneno de L. obliqua se han descrito tres actividades: una activadora de protrombina, otra de factor $X$ y una última activadora de fosfolipasa $\mathrm{A} 2$-like (8).
Antes de disponerse del antiveneno, el tratamiento estuvo enfocado al uso de los antifibrinolíticos; hoy día, sin embargo, con la disposición del suero antiLonomia desarrollado por el Instituto Butantan de Brasil, éste se constituye en el tratamiento de elección para los accidentes por orugas del género Lonomia, con las mismas especificaciones con las que se aplica el suero antiofídico. El resultado del tratamiento se puede monitorizar con las pruebas de coagulación $(19,20)$.

\section{Presentación de casos}

Caso 1: se trata de una mujer de 26 años, procedente de la vereda de San Nicolás (Nunchía, Casanare), de ocupación hogar. Ingresó al Hospital San Carlos de Bogotá el 4 de septiembre de 2000. La paciente manifestó que en la mañana del 30 de agosto fue picada en la mano por un gusano negro con 'cachitos'; inmediatamente presentó dolor en todo el miembro superior y cefalea que cedió con un analgésico común. La paciente llegó a la consulta con las orugas causantes del accidente, las cuales fueron identificadas posteriormente como L. achelous (figuras 1 y 2).

El 1 de septiembre presentó sangrado gingival y hemoptisis leve, por lo que viajó al Hospital de Yopal, en donde fue hospitalizada. El 2 de septiembre presentó sangrado gingival y disminución del volumen urinario. Los exámenes paraclínicos mostraron hemoglobina de $9,3 \mathrm{~g} / \mathrm{dl}$, hematocrito de $25 \%$, creatinina de $9 \mathrm{mg} / \mathrm{dl}$, BUN de $144 \mathrm{mg} / \mathrm{dl}$, tiempo de protrombina mayor de 31 segundos y tiempo parcial de tromboplastina mayor de 2 minutos; el recuento de plaquetas fue de $77.000 / \mu \mathrm{l}$. Fue remitida al hospital San Carlos de Bogotá por la necesidad de realizarle diálisis.

En el examen físico de ingreso, se encontró una paciente con tensión arterial de 130/90 mm Hg, frecuencia cardiaca de 92 por min, frecuencia respiratoria de 22 por min, ictérica, con aliento urémico, estigmas de sangrado gingival, estertores en ambos campos pulmonares y eritema en la mano izquierda. Por su estado de salud, ingresó a la unidad de cuidado intensivo con hemoptisis (figura 3 ), sangrado oral y saturación de oxígeno de $85 \%$. Se hizo una impresión diagnóstica de insuficiencia renal aguda, hemorragia alveolar 


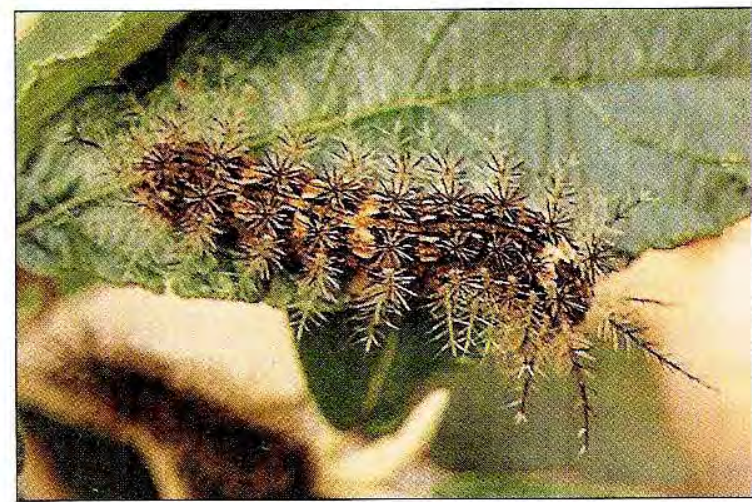

Figura 1. Oruga de Lonomia achelous, traida por la paciente del caso 1.

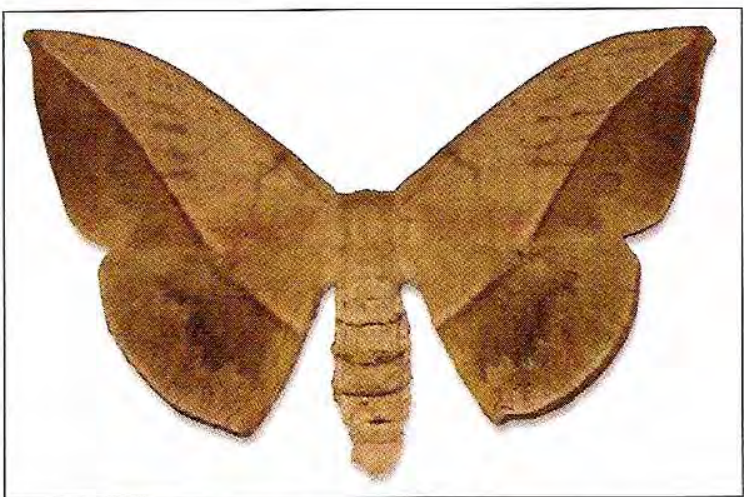

Figura 2. Adulto hembra de Lonomia achelous.

difusa, urgencia dialítica, CID y picadura de insecto.

Se realizaron exámenes paraclínicos y se encontró leucocitosis, neutrofilia, plaquetopenia severa (3.000/ $\mathrm{\mu l})$, anemia, tiempos de coagulación prolongados, pruebas de función renal y hepática elevadas, hipocaliemia corregida, acidemia metabólica, edema pulmonar y taquicardia sinusal. La ecografía abdominal mostró riñones grandes, indicativos de inflamación aguda y edema hepático. La paciente recibió transfusión de sangre y plaquetas y se le ordenó ventilación mecánica, diálisis y hemodiafiltración continua.

Se hicieron trámites para la consecución del suero anti-Lonomia fabricado en Brasil, pero, infortunadamente, no alcanzó a llegar. La paciente desarrolló un síndrome de respuesta inflamatoria sistémica, cuya manifestación más clara se apreció en la presentación de un cuadro de síndrome de

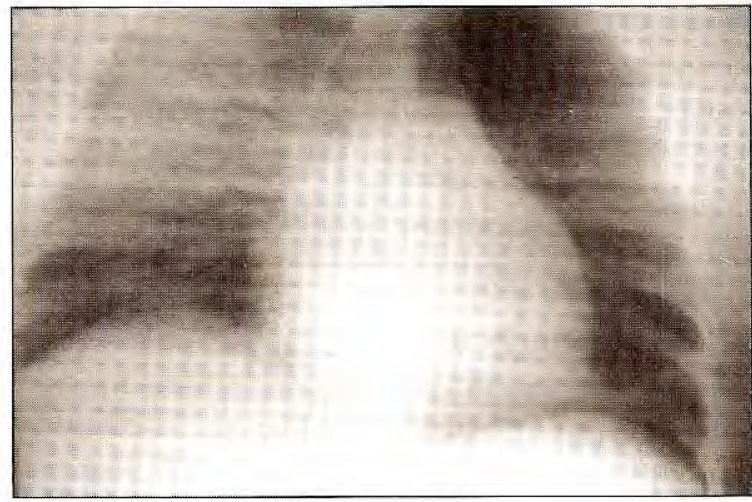

Figura 3. Placa de tórax del caso 1: se observan imágenes compatibles con sangrado alveolar difuso.

dificultad respiratoria aguda; requirió, además, grandes volúmenes de líquido y soporte respiratorio con presión positiva al final de la espiración y presión de soporte. Luego, desarrolló una disfunción orgánica múltiple a expensas de falla cardiaca, renal, respiratoria y hematológica. El deterioro fue progresivo, por lo cual se usaron inotrópicos en dosis máximas; sin embargo, entró en una disfunción orgánica múltiple con reanimación y la paciente falleció el 9 de septiembre.

Caso 2: se trata de una paciente de 21 años, procedente de la vereda Barquereña (San Luis de Palenque, Casanare). Ingresó al Hospital San Carlos de Bogotá el 20 de septiembre de 2000. La paciente refirió que el 16 de septiembre había sido picada por un churrusco en la parte interna del muslo derecho al recostarse contra un árbol. Presentó inicialmente dolor quemante y eritema local, seguido de náuseas y malestar general; a las 20 horas comenzó a presentar sangrado gingival y vaginal abundante, sin coágulos. La paciente consultó al Hospital de Yopal el 17 de septiembre por este cuadro, donde se hizo una impresión diagnóstica de accidente por Lonomia y fue remitida al Hospital San Carlos.

En el examen físico se encontró una paciente taquicárdica con palidez mucocutánea, ligero sangrado gingival, equímosis en el sitio de la picadura y sangrado vaginal. Los exámenes paraclínicos del 21 de septiembre mostraron dímero $D>2 \mathrm{fg} / \mathrm{ml}$ (valor normal: 0,008-0,135 fg/ml); glicemia, 92 mg/dl; BUN, 21 mg/dl; Na, 142,6 
$\mathrm{mEq} / \mathrm{l} ; \mathrm{K}, 2,97 \mathrm{mEq} / \mathrm{l} ; \mathrm{Cl}, 111 \mathrm{mEq} / \mathrm{L}$; hemoglobina, $6,69 \mathrm{~g} / \mathrm{dl}$ y hematocrito de $19 \%$; el recuento manual de plaquetas fue de $161.000 / \mu \mathrm{l}$, el producto de la degradación del fibrinógeno fue de $8 \mathrm{fg} / \mathrm{ml}$ (valor normal: 2-10 fg/m), tiempo de protrombina $>1$ min (control: $16,3 \mathrm{seg}$ ) y tiempo parcial de tromboplastina $>2$ min (control: $34,4 \mathrm{seg}$ ).

La impresión diagnóstica fue de CID secundaria a la picadura del insecto y hemorragia uterina anormal. Se le transfundieron 2 unidades de plasma fresco congelado y 1 unidad de glóbulos rojos empaquetados. El 22 de septiembre la paciente continuó con sangrado vaginal y sangrado gingival escaso. En la tarde se le aplicaron 2 ampollas de suero anti-Lonomia.

La paciente fue valorada por el Servicio de Ginecología que formuló estrógenos conjugados con el diagnóstico de hemorragia uterina anormal. Los exámenes paraclínicos antes de la administración del suero anti-Lonomia mostraron plaquetas $218.000 / \mu \mathrm{l}$, fibrinógeno indeterminable, tiempo de protrombina >1 min (control: $16 \mathrm{seg}$ ), tiempo parcial de tromboplastina de $49 \mathrm{seg}$ (control: $37 \mathrm{seg}$ ).

A partir del 24 de septiembre, la paciente no volvió a presentar sangrado; los tiempos de coagulación comenzaron a normalizarse y se le dió salida el 25 de septiembre.

\section{Discusión}

En el Hospital de Yopal se presentaron dos cuadros similares en un lapso menor de 20 dias, posteriores al contacto con orugas del género Lonomia. Se identificó $L$. achelous como agente causante de los dos accidentes; en el primer caso por identificación directa y en el segundo por clínica y antecedentes epidemiológicos. Ambos accidentes fueron remitidos al Hospital San Carlos de Bogotá y son los primeros descritos en el país.

El cuadro clínico que presentaron las dos pacientes fue similar a los descritos en la bibliografía sobre el tema; los exámenes paraclínicos confirman lo encontrado en otros estudios, con fibrinógeno bajo y tiempos de coagulación prolongados. La lesión renal descrita como una complicación del accidente por orugas del género Lonomia se corroboró en el caso 1 , mientras que en el caso 2 las pruebas de función renal fueron normales $(1,3)$.

El tratamiento de estos accidentes en Colombia presenta una nueva perspectiva con el desarrollo del suero anti-Lonomia del Instituto Butantan. Es fabricado con larvas de L. obliqua y mostró efectividad en el envenenamiento de la paciente del caso 2 , ocasionado presuntamente por $L$. achelous.

Después de esta presentación de los dos primeros casos en Colombia, es importante investigar los antecedentes de este tipo de accidentes en el país; es posible que se pueda tratar de los primeros casos que se presentan como resultado de la entrada al país de las orugas causantes de estos accidentes ( $L$. achelous) o que casos anteriores hayan pasado desapercibidos con otro tipo de diagnóstico (10). Igualmente, debe estudiarse la periodicidad y la estacionalidad del estado larvario de las polillas de las especies implicadas, ya que la fase de oruga (única causante de estas lesiones) tiene momentos definidos de ocurrencia, al parecer, después de las épocas lluviosas. Así mismo debe investigarse la posibilidad de que otras especies del género Lonomia en el país puedan producir estas lesiones y la efectividad del suero fabricado por el Instituto Butantan para tratar accidentes ocasionados por otras especies de Lonomia. La distribución geográfica de las especies de Lonomia involucradas en este tipo de accidentes y el establecimiento de medidas de prevención que disminuyan el riesgo de contacto de los pobladores con orugas de estas especies son, igualmente, prioridad para el estudio.

\section{Agradecimientos}

A Jaime Ramírez por la colaboración con material bibliográfico; a él y a la empresa BP: BP Exploration Colombia por la consecución del suero antiLonomia y a Magnolia Arango por su colaboración para establecer el diagnóstico.

\section{Bibliografía}

1. Duarte AC, Caovilla J, Lorini I, Lorini D, Mantovani $\mathbf{G}$, Sumida $\mathbf{J}$ et al. Insuficiencia renal aguda por acidentes com lagartas. J Bras Nefrol 1990;12:184-7.

2. Duarte AC, Crusius PS, Pires CA, Schilling MA, Wen FH. Intracerebral haemorrhage after contact with Lonomia caterpillars. Lancet 1996;348:1033. 
3. Wen FH, Cardoso JL, Olmos RD, Almeida J, Viana RP, Martinez APP. Hemorrhagic syndrome and acute renal failure in a pregnant woman after contact with Lonomia caterpillars: a case report. Rev Inst Med Trop S Paulo 1998;40:119-20.

4. Arocha-Piñango CL. Fibrinolisis producida por contacto con orugas: comunicación preliminar. Acta Cient Venez 1967;18:136-9.

5. Fraiha $H$, Ballarini AJ, Leao RNQ, Costa JR, Dias, LB. Síndrome hemorrágica por cơntacto com lagartas de mariposa (Lepidoptera, Saturniidae) En: Instituto Evandro Chagas: 50 anos de contribuição as ciencias biológicas e a Medicina tropical. Belem: Fundaçao Servicios de Saude Publica; 1986. p. 811-20.

6. Couppie P, Marty C, Sainte-Marie D, Pradinaud R. Poisonous caterpillars in French Guyana: 5 cases. Ann Dermatol Venereol 1998;125:489-91.

7. Burdmann EA, Antunes I, Saldanha LB, Abdulkader RCMR. Severe acute renal failure induced by the venom of Lonomia caterpillars. Clin Nephrol 1996;46:337-9.

8. Arocha-Piñango $\mathrm{CL}$, Marval E, Guerrero B. Lonomia genus caterpillar toxins: biochemical aspects. Biochimie 2000;82:937-42.

9. Lemaire, C. Revision du genre Lonomia Walker. Ann Sc Ent FR (n.S). 1972;8:767-861.

10. Hommel D, Bouchareine L, Hulin A. Envenimation par une chenille de lépidoptere: Lonomia achelous Revue de la litérature, a propos de deux cas en Guyane Française. Sem Hop Paris 1995;71:9-12.

11. Arocha-Piñango $C L$, Layrisse $M$. Fibrinolysis produced by contact with a caterpillar. Lancet 1969 ; i: $810-2$.

12. Arocha-Piñango $C L$. Venenos de invertebrados $y$ coagulación. En: Hematología geográfica. Volumen IV.
Enciclopedia Iberoamericana de Hematologia, Salamanca, España: Ediciones Universal de Salamanca; 1992. p. 670-5.

13. Duarte AC, Caovilla JJ, Hermann F, Renner LO. Hemorragia géngivo-jugal pós-contato com Lonomia obliqua. Rev Med Hosp Sao Vicente de Paulo 1997; 9:52-4.

14. Marval E, Guerrero B, Arocha-Piñango CL. The action of Lonomia achelous caterpillar venom on some blood coagulation and fibrinolysis parameters of the rabbit. Toxicon 1999;37:1491-504.

15. Lorini L. Lagarta - Lonomia obliqua. En: Nicollela A, Barros E, Torres JB, Marques MG, editores. Acidentes com animais peçonhentos. Consulta rápida. Porto Alegre: Hospital de Clínicas de Porto Alegre; 1997. p. 51-3.

16. Arocha-Piñango CL, Bosch NB, Torres A, Goldstein C, Nouel A, Arguello A, et al. Six new cases of a caterpillar-induced bleeding syndrome. Thromb Haemostas 1992;67:402-7.

17. López M, Gil A, Arocha-Piñango CL. The action of Lonomia achelous caterpillars venom on human factor V. Thromb Res 2000;98:103-10.

18. Duarte A, Walter G, Barros E. Lonomia obliqua. En: Nicollela A, Barros E, Torres JB, Marques MG, editores. Acidentes com animais peçonhentos. Consulta rápida. Porto Alegre: Hospital de Clinicas de Porto Alegre; 1997. p. 163-8.

19. Dias da Silva W, Rocha ACM, Gonçalves LR. Development of an antivenom against toxins of Lonomia obliqua caterpillars. Toxicon 1996;34:1045-9.

20. Wen FH. Revisão dos aspectos terapeuticos em acidentes por Lonomia sp. En: Nicollela A, Barros E, Torres JB, Marques MG, editores. Acidentes com animais peçonhentos. Consulta rápida. Porto Alegre: Hospital de Clinicas de Porto Alegre; 1997. p.169-72. 